\title{
Article
}

\section{The temporal pattern of recovery in directional dynamic stability post-football specific fatigue}

Rhodes, David, Alexander, Jill and Greig, Matt

Available at http://clok.uclan.ac.uk/37439/

Rhodes, David ORCID: 0000-0002-4224-1959, Alexander, Jill ORCID: 00000002-6492-1621 and Greig, Matt (2021) The temporal pattern of recovery in directional dynamic stability post-football specific fatigue. Journal of Sport Rehabilitation . ISSN 1056-6716

It is advisable to refer to the publisher's version if you intend to cite from the work.

For more information about UCLan's research in this area go to http://www.uclan.ac.uk/researchgroups/ and search for <name of research Group>.

For information about Research generally at UCLan please go to http://www.uclan.ac.uk/research/

All outputs in CLoK are protected by Intellectual Property Rights law, including Copyright law. Copyright, IPR and Moral Rights for the works on this site are retained by the individual authors and/or other copyright owners. Terms and conditions for use of this material are defined in the policies page.

\section{CLoK}

Central Lancashire online Knowledge www.clok.uclan.ac.uk

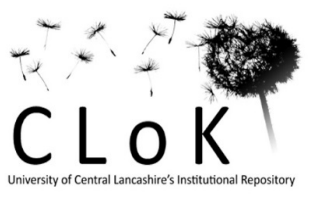


1 Title

2 The temporal pattern of recovery in directional dynamic stability post-football specific fatigue.

3

\section{Authors}

5 Dr David Rhodes $\mathrm{PhD}^{1}$; Jill Alexander²; Dr Matt Greig $\mathrm{PhD}^{3}$.

6

\section{$7 \quad$ Affiliations}

$8{ }^{1}$ Institute of Coaching and Performance (ICaP), School of Sport and Health Sciences, University of 9 Central Lancashire, Preston, UK.

$10{ }^{2}$ Sport, Nutrition and Clinical Sciences, School of Sport and Health Sciences, University of Central 11 Lancashire, Preston, UK.

$12{ }^{3}$ Department of Sport and Physical Activity, Edge Hill University, Ormskirk, Lancashire, L39 4PQ.

\section{Corresponding Author}

15 Dr David Rhodes

16 DRhodes2@uclan.ac.uk

ORCID - 0000-0002-4224-1959

\section{Conflict of Interest}

There are no conflicts of interests to report.

\section{Funding}

None to declare. 


\section{Abstract}

Background: Rising injury rates within football require further understanding of aetiological risk factors associated with lower limb injury.

Aim: The aim of the present study was to examine the temporal pattern of recovery of directional dynamic stability measures post-football specific fatigue.

Methods: Eighteen male elite footballers completed baseline assessments of directional dynamic stability measures (Overall Stability Index (OSI); Anterior-Posterior stability (A-P); Medial-Lateral Stability (M-L) on level 1 of the Biodex Stability System (BSS). Post SAFT ${ }^{90}$ measures were repeated immediately, $+24 \mathrm{hrs},+48 \mathrm{hrs}$ and $+72 \mathrm{hrs}$. Main effects for recovery time and direction of stability were supplemented by regression modelling to describe the temporal pattern of recovery.

Results: Significant main effects for time were identified for all directions of stability (OSI, A-P and M-L) up to $+48 \mathrm{hrs}$ post exercise $(p \leq 0.05)$. The quadratic pattern to temporal recovery highlights a minima of 37.55 - 38.67hrs and maxima of 75.09 - 77.33hrs. Additionally, a main effect for direction of stability was observed, with significant differences identified between A-P and M-L stability at all timepoints $(p \leq 0.001)$.

Conclusions: Reductions in directional dynamic stability $+48 \mathrm{hrs}$ post fatigue highlight implications for training design, recovery strategies and injury management for performance practitioners. Interestingly, A-P stability has been highlighted as being significantly reduced compared to M-L stability at all time points, regardless of the fatigue exposure. Practitioners should consider the reduction of stability in this plane in relation to common mechanisms of injury in the knee to inform injury risk reduction strategies.

Keywords: Performance; Recovery; Stability; Soccer; Screening. 


\section{INTRODUCTION}

Evidence highlights proprioception and fatigue as two key aetiological risk factors associated with lower limb injury. ${ }^{1,2,3}$ The operational definition of proprioception is the body's ability to sense movements within joints and to have a knowledge of where these joints are in relation to space. ${ }^{4}$ Interpretation and quantification can cause confusion amongst applied practitioners, as measures, tests and exercise prescription relate to the effected output and do not quantify the physiological process. ${ }^{5,6}$ Although it is accepted that the effected output relates to how efficient this neuromuscular process is, future evidence requires clarity on what is being measured to inform injury risk reduction strategies. Debate exists with regards to the effect of fatigue on proprioceptive ability with contrasting views of whether it has any effect at all. ${ }^{7}$ Within this present body of work the extent of fatigue and type of training is not well described. Furthermore, variation of the quantification of proprioceptive output exists between studies in terms of joint position sense, balance or dynamic stabilisation. ${ }^{2,7,8}$ Thus, drawing varying conclusions in response to a fatigue exposure and reasons reduction in performance. Arguably, when relating these findings to injury risk, the effected output is often the focus, relating strongly to dynamic stability responses.

Dynamic stability can be quantified through various subjective or objective means, such as single leg landing, single leg-hop and hold, Y-balance, single leg stand; to name a few., ${ }^{3,1,9}$ Commonly when objectively quantifying dynamic stability, literature has focussed on determining an overall stability index (OSI) and refrained from deeper discussion surrounding directional stability. ${ }^{7,10}$, Further understanding of directional dynamic stability response (DDS) may provide practitioners with further insight for conditioning and injury risk reduction strategies. Dynamic stabilisation of the lower limb is affected by muscle response, and as such, poor muscle response may increase injury risk. ${ }^{11}$ Movement patterns performed in football are multi-planar and it is not unreasonable to suggest directional output is important to observe and quantify.

Eccentric loading experienced during game play during such movements is reported to be the most damaging type of muscle contraction that an athlete can be exposed to, resulting in potential detrimental effects on strength. ${ }^{12,13,14}$ Consequently, exposure to exhaustive eccentric loading may result in reductions in dynamic stability performance, ${ }^{2,3}$ demonstrating the influence of muscle response on dynamic stabilisation. ${ }^{15}$. Conclusions drawn from the literature suggest that the fatiguing nature of eccentric loading results in an inhibition of the intrafusal fibres of the muscle spindles and golgi tendon organs (GTO). ${ }^{16,17}$ This inhibition of the neuromuscular pathway may affect strength response or result in an electromechanical delay (EMD) in the neuromuscular pathway, thus impeding dynamic stability output. ${ }^{18}$ Either response would result in increased injury risk, due to exposure of the joint to increased loading or abnormal movement patterns. Several studies acknowledge the effect of fatigue on eccentric 
strength of the hamstrings, with the consensus that eccentric strength reduces significantly. ${ }^{12,13,14}$ It is suggested that the implications of this are wider. During functional performance the hamstring is a key muscle in stabilisation of the knee, most notably anterior translation and rotation. ${ }^{19,20}$ Consequently, these two movement patterns, through differing planes, are largely associated with anterior cruciate ligament (ACL) injury. ${ }^{6,21,22}$ Consequently, implications may also be observed inferiorly to the knee, potentially affecting the ankle joint control mechanisms. Failure to identify the effect of fatigue on directional dynamic stability does not provide the practitioner with enough detail to inform injury risk reduction strategies in the lower limb.

Reduced dynamic stability has been associated with common lower limb injuries often seen in elite footballers, predominantly associated with the ankle (lateral ankle sprains) and knee (ACL). ${ }^{21,23}$ Interestingly, current evidence has reported a large increase in the number of ACL injuries sustained within elite male footballers and the detrimental effect these injuries can have on a player's career. ${ }^{19,22,23}$ Both lateral ankle sprains and knee injuries are associated with multi-planar movements when the injury is sustained. ${ }^{22,23,24}$ Analysis of an athlete's functional directional dynamic stability is essential for determining injury risk. ${ }^{9}$ Evidence surrounding the fatigue effect on dynamic stabilisation focusses primarily on an immediate response ${ }^{1,5}$ and falls short of successfully analysing the resultant temporal pattern. This approach fails to consider the context of contemporary elite football, where demand is placed on frequency and subsequent congestion of fixtures and training. Literature clearly highlights the detrimental effect of fixture congestion on injury ${ }^{25,26}$. Subsequently further identification of the temporal pattern of directional dynamic stabilisation post-football specific fatigue, would inform training design and injury risk reduction strategies. Therefore, the aim of the present study is to quantify the temporal pattern of recovery in directional dynamic stabilisation of elite footballers for $72 \mathrm{hrs}$ post a simulated specific fatigue protocol.

\section{METHODS}

\section{Participants}

From an available squad of twenty-four, eighteen elite male football players took part (age: $22.94 \pm 4.57$ years, height: $185.3 \pm 4.2 \mathrm{~cm}$; body mass: $75.91 \pm 6.38 \mathrm{~kg}$ ). A minimum sample size was based on sixteen players who met the inclusion / exclusion criteria of; no history of previous lower limb injury in the last 6 months and highlighted by the clubs medical team as having no mechanical or functional instability in the knee or ankle at time of testing. ${ }^{9}$ Players included were also free from systemic or vestibular disorders known to impair cutaneous sensation of balance. ${ }^{7}$ In total, eight players were excluded from partaking in the study due to: injury $(n=2)$, playing 
position (goalkeepers' $n=3$ ), unavailable due to being on loan to another club $(n=3)$. All players provided written informed consent in accordance with Department and Faculty Research Ethics committees at the host University (SPA-REC-2014-334), and in accordance with the Declaration of Helsinki (2013).

\section{Experimental Design}

Players completed a familiarisation trial 7 days prior to testing to negate potential learning effects, ${ }^{27}$ which included the football Aerobic Field Test $\left(\mathrm{SAFT}^{90}\right) \operatorname{protocol}^{28}$ and the directional dynamic stability (DDS) testing on the BSS (Medical Systems, Shirley, NY.) Subsequently, the testing session also included elements of the $\mathrm{SAFT}^{90}$ as part of the pre-exercise warm-up, and trial repetitions of the DDS testing on the BSS. All testing was completed between 13:00 and 17:00 hrs to account for the effects of circadian rhythm and in accordance with regular competition times. ${ }^{29}$ All trials were completed on the dominant lower limb, identified by their favoured kicking foot, based on non-contact musculoskeletal injury epidemiology. ${ }^{30}$

All testing was completed on the same BSS at testing level 1. The BSS (Biodex Medical Systems, Shirley, NY) is an unstable platform that can tilt up to $20^{\circ}$ in any direction, with the stability of the platform determined by the level by which it is set ranging from 1 (most unstable) to 12 (most stable). ${ }^{31}$ Research has shown that the most appropriate level for an elite footballer is level 1, with ICC repeatability scores indicated to be $0.85 .{ }^{8,32}$ Players were asked to complete 10 minutes of the SAFT ${ }^{90}$ protocol as a warm up followed by directed dynamic stretching focussed on the quadriceps, hamstrings, gluteal muscles and gastrocnemius. The $\mathrm{SAFT}^{90}$ was utilised within the study as it is a free running protocol that replicates the physiological and mechanical demands experienced during game play. ${ }^{28}$ Over a 20m distance, players move through a series of cones and poles, alternating between side steps, backwards running, accelerations and decelerations with varying intensities, which are prompted by audio cues. The 15-min activity profile is repeated six times to formulate the 90 mins, with players having a 15-min half time break, where they are directed to sit, as they would in normal game play. The activity profile is performed in a randomised and intermittent fashion and incorporates 1269 changes in speed and 1350 changes in direction over a 90 -min period. ${ }^{13}$

Pre-exercise, all players completed the BSS testing, which comprised of 3 trials of 20-seconds completed on stability level 1. Once completed metrics of Overall Stability Index (OSI), AnteriorPosterior (A-P) and Medial-Lateral (M-L) were observed and noted. These metrics were calculated by the BSS software and based on the amount of tilt in degrees of the foot platform during the trial. A low index score indicated high stability and high score a low level of stability. All testing on the BSS was 
completed barefoot, due to the effect footwear can have on kinematics of the foot and muscle activity in the lower limb. ${ }^{33}$ The BSS was setup in accordance with previous literature. ${ }^{8}$ They then stood on the platform in full extension with their dominant limb with their foot in the centre of the platform. The feedback screen was set at eye level and the players were asked to look at the screen, this was set as such to avoid any unwanted head movement and avoid vestibular distraction. ${ }^{8}$ Once set the players were then asked to adjust their standing foot to a comfortable position, while the marker on the feedback screen maintained a central position, once done and comfortable the platform was locked into a stable position and the players foot position was recorded. Once recorded the foot position remained consistent through each trial throughout the entire testing period. In between each trial players were told to weight bear through the contralateral limb to minimise the effect of fatigue when testing. In cases where players lost their balance, they were told to use the contralateral limb to stabilise themselves by placing it at the back corner of the BSS and were only encouraged to use the handrails if they completely lost balance. Figure 1 provides a representation of the testing set-up.

$$
\text { ***insert figure } 1 \text { here } * * *
$$

Following completion of the SAFT ${ }^{90}$ the standardised BSS testing was completed immediately. Further trials were completed following the same protocol at $+24 \mathrm{hrs},+48 \mathrm{hrs}$ and $+72 \mathrm{hrs}$ post $\mathrm{SAFT}^{90}$ in order to monitor the temporal pattern of recovery in DDS performance. ${ }^{14}$ Between trials players were reminded to refrain from exercise and to maintain a normal diet.

\section{Statistical Analysis}

The directional stability output was analysed for each 20 -second trial and an average score across the three trials was taken for analysis. Directional stability scores were identified for OSI, anterior-posterior stability (A-P) and medial-lateral stability (M-L). Each directional stability variable was determined pre-exercise, immediately post-exercise, and then at $+24-,+48$ - and +72 -hrs after exercise.

A univariate repeated measures general linear model was used to quantify main effects for recovery duration post-exercise and DDS. Interaction effects were quantified, and significant main effects in recovery duration were explored using post-hoc pairwise comparisons with a Bonferonni correction. The assumptions associated with the statistical model were assessed to ensure model adequacy. To assess residual normality for each dependant variable, $\mathrm{q}-\mathrm{q}$ plots were generated using stacked standardised residuals. Scatterplots of the stacked unstandardised and standardised residuals were utilised to assess the error of variance associated with the residuals. Mauchly's test of sphericity was completed for all dependent variables, with a Greenhouse Geisser correction applied if the test was significant. Partial eta squared $\left(\eta^{2}\right)$ values were calculated to estimate effect sizes for all significant 
main effects and interactions. As recommended in literature ${ }^{34}$, partial eta squared was classified as small (0.01-0.059), moderate (0.06-0.137), and large $(>0.138)$.

The temporal pattern of changes in each directional dynamic stability variables over the $72 \mathrm{hr}$ data collection period was examined using a regression analysis. Linear and quadratic polynomial models were applied, with the optimum fit determined by the strength of the correlation coefficient (r). Where a quadratic regression analysis represented the best fit, the regression equation was differentiated with respect to time to elicit the time (post-exercise) at which the data reached maxima (or minima). All statistical analysis was completed using PASW Statistics Editor 26.0 for windows (SPSS Inc, Chicago, USA). Statistical significance was set at $P \leq 0.05$, and all data are presented as mean \pm standard deviation.

\section{RESULTS}

Figure 2 summarises the effects of the exercise protocol and the temporal pattern of recovery on directional dynamic stability, for OSI, A-P and M-L. There was a significant main effect of direction $\left(F=34.20, p<0.001, \eta^{2}=0.21\right)$ and time $\left(F=10.54, p=<0.001, \eta^{2}=0.14\right)$. Significant differences were displayed between all directions of stability represented as $p \leq 0.05$. Pre-exercise directional stability values were significantly lower (pre to post: $p \leq 0.05$; pre to $+24 \mathrm{hrs}$ and pre to $+48 \mathrm{hrs} p \leq$ $0.001)$, no significant difference was indicated pre to $+72 \mathrm{hrs}(p>0.05)$. There was no direction $x$ time interaction $\left(F=0.49, p=0.38, \eta^{2}=0.12\right)$.

$$
\text { ***insert figure } 2 \text { here } * * *
$$

With the data set collapsed to consider the temporal pattern of recovery for each direction of stability, all directions displayed a significant effect of time (OSI: $F=3.98 ; p=0.005, \eta^{2}=0.16$; A-P: $F=2.81$; $p=0.03, \eta^{2}=0.12 ;$ M-L: $\left.F=5.19 ; p=0.001, \eta^{2}=0.20\right)$. OSI and M-L directional stability displayed significant increases in stability scores up to $+48 \mathrm{hrs}$ when compared to pre exercise scores $(p \leq 0.05)$. A-P stability displayed no significant difference between pre and immediately post exercise stability $(p$ > 0.05). Although displayed significant increases in stability values pre to +24 hrs and pre to $+48 \mathrm{hrs}$ post exercise $(p \leq 0.05)$. Each direction of stability also indicated significant reductions in stability scores from $+24 \mathrm{hrs}$ to $+72 \mathrm{hrs}(p \leq 0.05)$. Significant differences in stability scores were also found between each directional measure $(p \leq 0.001)$. It was found that OSI stability scores were significantly higher that A-P and M-L $(p \leq 0.001)$ and A-P was significantly higher than M-L $(p \leq 0.001)$. Mean stability scores also highlighted that A-P stability scores at each time point were higher than M-L stability scores $(14-25 \%)$. 
259 The relationship between directional dynamic stability and post-exercise recovery duration was best 260 represented as a quadratic polynomial function $(r=\geq 0.86)$. The differentiated regression equations 261 yielded a minima between $37.55-38.00 \mathrm{hrs}$ (OSI: 37.55hrs $(\mathrm{y}=-0.0011 \mathrm{x} 2+0.0826 \mathrm{x}+2.5303)$; A-P: $26238 \mathrm{hrs}(\mathrm{y}=-0.0007 \mathrm{x} 2+0.0532 \mathrm{x}+1.9633) ;$ M-L: 38.67hrs $(\mathrm{y}=-0.0006 \mathrm{x} 2+0.0464 \mathrm{x}+1.5125))$ and maxima between 75.09 - 77.33hrs (OSI: 75.09hrs; A-P: 76hrs; M-L: 77.33hrs). This would result in a predicted return to baseline values of up to $77.33 \mathrm{hrs}$.

\section{DISCUSSION}

The aim of the present study was to investigate the temporal pattern of recovery in directional dynamic stability in elite male footballers for up to 72-hrs post a simulated football specific fatigue protocol. Research in this area is limited, as it predominantly focuses on the immediate acute effect of fatigue and does not differentiate measures based on the direction of stability. ${ }^{1,2,3,32}$ It was observed that completion of the fatigue protocol induced an average $22 \%$ - 30\% reduction in directional dynamic stability scores (OSI, A-P and M-L) when compared to pre-fatigue measures. These findings are consistent with previous research that analysed the effects of fatigue on eccentric hamstring strength. ${ }^{12,13,14}$ Current findings, in agreement with previous studies report positive correlations between hamstring function and directional dynamic stability. ${ }^{9}$ Main findings within this body of work indicate significant differences between pre exercise measures and $+48 \mathrm{hrs}$ for all directions of stability, with no differences observed at $72 \mathrm{hr}+$. Interestingly, no significant differences were observed for A-P stability when compared to pre fatigue measures, despite a $22 \%$ average increase in scores. Significant reductions in stability scores for all directions were observed between $24 \mathrm{hr}+$ and $72 \mathrm{hr}+$, highlighting a period of recovery. This said, a 14-19\% increase in stability scores still existed at $72 \mathrm{hr}+$ when compared to pre-fatigue values. Further analysis via polynomial regression indicated that mean stability values took up to $77.33 \mathrm{hrs}$ to return to pre-exercise levels, with variations of recovery within specific directions of stability metrics. The present findings are based on a cohort of elite footballers, where group averages are observed. In practice it is important to consider individual responses to fatigue and their temporal pattern, as these will differ between players. Future research should consider these factors to bridge the gap between academia and applied practice.

Controversy exists within recent evidence indicating that sustaining knee injuries, such as anterior cruciate ligament (ACL), may not be related to fatigue. ${ }^{35}$ This contradicts previous evidence, where fatigue has been highlighted as a key aetiological factor to sustaining these injuries. ${ }^{36}$ It is important to note that the research by Doyle et al., (2019) only observes the stage of the season and period of the game when the injuries were sustained. Concluding that there were no specific periods of the season or difference between halves of the game when these injuries were sustained, thus discounting a fatigue 
effect. Although, this may discount the acute fatigue responses (in game), there is no indication within the study of the players readiness to play. Prompting the question of whether sustaining this injury was a result of accumulative fatigue during a fixture congested period or an increased training load exposure. ${ }^{12,14}$ Understanding of the temporal pattern of fatigue in relation to directional dynamic stability provides further insight into the recovery patterns of footballers in relation to key aetiological factors associated with injury. Particularly useful in the current modern game where COVID-19 has impacted on recovery time due to condensed fixture schedules, reduced preparation and training. It is important to note that injury risk is multi factorial and this is only one piece of the injury risk paradigm. Present findings fail to consider a multifactorial model and future research should consider multiple outcome measures utilised in the field to quantify fatigue effect. They should carefully relate to biomechanical, physiological and psychological measures.

ACL injuries sustained in footballers are commonly associated with an increased anterior shearing force. Resulting in excessive load being exerted through the ACL. ${ }^{19,20}$ It is suggested that decreased functional strength of the hamstrings may have an impact on directional dynamic stability performance, ${ }^{9}$ and with the addition of acute fatigue exposure or insufficient recovery this risk could be heightened. Combined with the additional rotational load, potentially creating further damage to major joint structures, these injuries can result in significant time loss for the athlete. ${ }^{19,22,23}$ The hamstrings are a key muscle group providing support to the knee joint through these movement patterns, best explained by understanding its functional anatomical role in performance. ${ }^{20}$ Future work should consider quantifying muscle activity in relation to dynamic stability output, with the use of electromyography. The present study identifies significantly elevated A-P stability scores throughout all time points compared to M-L (14-25\%), which potentially indicates the vulnerability of the ACL to increased anterior load exposure during performance. This further acknowledges the vulnerability of the structure and recent evidence to suggest that injury occurrence may not solely be attributed to fatigue. ${ }^{35}$ Further research is required and should consider the effect of training load, accumulated fatigue or readiness to play when analysing injury incidence in game play or within periods of high occurrences of ACL involvement.

It is important to note that the post-exercise response displayed in the present study highlighted an increase in directional dynamic stability scores up to a predicted $77 \mathrm{hrs}$ post exercise (Figure 2), potentially increasing injury risk. This is accompanied by increases in M-L stability. Providing support to suggest that eccentric loading experienced during football specific activity can result in an inhibition of the intrafusal fibres of the muscle spindles and GTO. ${ }^{16,17}$ Thus, reducing muscle response to provide dynamic stabilisation. The reduction in stabilisation experienced, potentially as a result of reduced eccentric hamstring function, may have implications to other joints within the lower limb. It is important to note the reduced function of the hamstrings as discussed may not be the only contributory 
factor to reductions in dynamic stabilisation. These links are suggestive based on previous findings, consideration of the functional anatomy and results reported within the present study. Further research should consider the effect and influence of other muscle groups within the lower limb and their influence on lower limb dynamic stability. Consideration should also be given to the electromyographic response of varying muscle groups within the lower limb in response to fatigue and directional dynamic stability.

Literature has indicated the acute detrimental effect of fatigue on dynamic stability ${ }^{1,2,3,32}$ with directional stability being identified as a key aetiological factor associated with common non-contact lower limb musculoskeletal injuries. ${ }^{6,21,22}$ It is important to contextualise the meaning of OSI, A-P and M-L stability. Previous research has heavily focussed on only OSI scores when quantifying dynamic stability. ${ }^{7,10}$ It is suggested however that further insight into function to inform injury risk may be gained from observing the planar outputs of A-P and M-L directional stability. These specific movement patterns can be related to the injury mechanism during functional performance. Consideration of the mechanisms associated with common joint injuries sustained at the knee and ankle indicate that injuries sustained at these joints may be associated with multi planar movement patterns. ${ }^{22,23}$ The findings of the present study highlight the differing temporal pattern of recovery of directional dynamic stability (OSI, A-P and M-L) post-football specific fatigue, displaying a return to baseline at $77.33 \mathrm{hrs}$. In addition, this identifies the differential response of A-P to M-L dynamic stability when quantified on the BSS. Interestingly the detrimental effect of exercise continues for $38.67 \mathrm{hrs}$ post-football specific fatigue. Consequently, these findings provide considerations for training design, player monitoring and injury risk reduction strategies in elite football settings.

\section{Conclusions}

Directional dynamic stability of OSI, A-P and M-L were shown to significantly deteriorate up to $48 \mathrm{hrs,}$ as a result of football specific fatigue. Quadratic polynomial regression modelling suggest that mean scores only truly returned to baseline at $77.33 \mathrm{hrs}$ and continued to decline post fatigue exposure up to $38.67 \mathrm{hrs}$. Regardless of time points, A-P stability measures were shown to be significantly elevated compared to M-L, indicating poorer stability performance through this plane. These findings provide important considerations for sports performance practitioners with regards injury risk reduction, recovery interventions and training design. Careful consideration must be given to the implications of these findings and their association with the mechanism of common lower limb injuries sustained in football. Injury risk reduction strategies, conditioning or rehabilitation of the athlete post injury should consider specific directional stability training when the athlete is fatigued. 
1. Changela PK, Selvamani K, Ramaprabhu. A Study to Evaluate the Effect of Fatigue on Knee Joint Proprioception and Balance in Healthy Individuals. Int J Scientific Res Publications 2012;2: $1851-1857$.

2. Melnyk M, Gollhofer A. Submaximal Fatigue of the Hamstrings Impairs Specific Reflex Components and Knee Stability. Knee Surg Sports Traumatol Arthrosc, 2007;15: 525 - 532.

3. Letafatkar K, Alizadeh MH, Kordi MR. The Effect of Exhausting Exercise Induced Muscular Fatigue on Functional Stability. J Social Sci, 2009;4: 416 - 422.

4. Torres R, Duarte JA, Cabri JMH. An Acute Bout of Quadriceps Muscle Stretching has no Influence on Knee Joint Proprioception. J Human Kinetics 2012;34:33 - 39.

5. Ribeiro F, Santos F, Goncalves P, Oliveira J. Effects of volleyball match induced fatigue on knee joint position sense. Eur J Sports Sci 2008;8: 397 - 402.

6. Alentorn-Geli E, Myer GD, Silvers HJ, Samitier G, Romero D, Lazaro-Haro C, Cugat R. Prevention of Non-Contact Anterior Cruciate Ligament Injuries in Soccer Players. Part 1: Mechanisms of Injury and Underlying Risk Factors. Knee Surg Sports Traumatol Arthrosc 2009; 17: $705-729$.

7. Gioftsidou A, Malliou P, Pafis G, Beneka A, Tsapralis K, Sofokleous P, Kouli O, Roka S, Godolias G. Balance Training Programs for Soccer Injuries Prevention. J Human Sport Ex 2012;7: 639 - 647.

8. Arifin N, Osman NAA, Abas WAB. Intrarater Test-Retest Reliability of Static and Dynamic Stability Indexes Measurement Using the Biodex Stability System During Unilateral Stance. J App Biomech 2014;30: 300 - 304.

9. Rhodes D, Leather M, Birdsall D, Alexander J. The Effect of Proprioceptive Training on Directional Dynamic Stabilisation. J Sports Rehab 2020; In Press Accepted.

10. McHugh MP, Tyler TF, Mirabella MR. The Effectiveness of a Balance Training Intervention in Reducing the Incidence of Non-Contact Ankle Sprains in High School Football Players. Am J Sports Med 2007;35: 1289 - 1294.

11. De Ste Croix MBA, Priestly AM, Lloyd RS, Oliver JL. ACL injury risk in elite female youth soccer: Changes in neuromuscular control of the knee following soccer-specific fatigue. Scand J Med \& Sci Sports 2015;25: 531 - 538.

12. Greig, M. The influence of soccer-specific fatigue on peak isokinetic torque production of the knee flexors and extensors. Am J Sports Med 2008;36: 1403-1409.

13. Small K, McNaughton LR, Greig M, Lovell R. The effects of multidirectional soccer-specific fatigue on markers of hamstring injury risk. J Sci Med Sport 2009;13: 120-125.

14. Rhodes D, McNaughton L, Greig M. The Temporal Pattern of Recovery in Eccentric Hamstring Strength Post Soccer Specific Fatigue. Res Sports Med 2018;27: 339 - 350. 
15. Hody S, Croisier JL, Bury T, Rogister B, Leprince P. Eccentric Muscle Contractions: Risks and Benefits. Front in Phys 2019;10: 1 - 18.

16. Chen HL, Nosaka K, Chen TC. Muscle Damage Protection by Low-Intensity Eccentric Contractions Remains for 2 Weeks but not 3 Weeks. Eur J Appl Physiol 2012;112: 555 - 565.

17. Opar D, Williams M, Timmins R, Hickey J, Duhig S, Shield A. Eccentric Hamstring Strength During the Nordic Hamstring Exercises is a Risk Factor for Hamstring Strain Injury in Elite Australian Football: A Prospective Cohort Study. Br J Sports Med 2014;48: 647 - 648.

18. Conchola E C, Thompson B J, Smith D B. 2013. Effects of Neuromuscular Fatigue on the Electromechanical Delay of the Leg Extensors and Flexors in Young and Old Men. European J of App Physiol, 2013; 113(9): 2391 - 2399

19. Bryant AL, Clark RA, Pua YH. Morphology of Hamstring Torque-Time Curves following ACL Injury and Reconstruction: Mechanisms and Implications. J Orthopaedic Res 2011;29: $907-914$.

20. Arnason SM, Birnir B, Gudmundsson G, Briem K. Medial Hamstring Muscle Activation Patterns are Affected 1-6 Years after ACL Reconstruction Using Hamstring Autograft. Knee Surg Sports Traumatol Arthrosc 2014;22: 1024 - 1029.

21. Walden M, Hagglund M, Magnusson H, et al. Anterior cruciate ligament injury in elite football: A prospective three-cohort study. Knee Surg Sports Traumatol, Arthrosc 2011;19: 11-19.

22. De Ste Croix MBA, Elnagar YO, Iga J, James D, Ayala F. Electromechanical Delay of the Hamstrings During Eccentric Muscle Actions in Males and Females: Implications for NonContact ACL Injuries. J Electromyography Kinesiology 2015;25: 901 - 906.

23. Walden M, Hagglund M, Magnusson H, Ekstrand J. ACL injuries in Men's Professional Football: A 15-year Prospective Study on Time Trends and Return to Play Rates Reveals only 65\% of Players Still Play at the Top level 3 years after ACL Rupture. Br J Sports Med 2016;50: $744-750$.

24. Walden M, Hagglund M, Ekstrand J. Time-Trends and Circumstances Surrounding Ankle Injuries in Men's Professional Football: An 11 year Follow up of the UEFA Champions League Injury Study. Br J Sports Med 2013;47: 748 - 753.

25. Dupont G, Nedelec M, McCall A. Effect of 2 Soccer Matches in a Week on Physical Performance and Injury Rate. Am J of Sports Med 2010;38: 1752 - 1758.

26. Dellal A, Lago-Penas C, Rey E. The Effects of a Congested Fixture Period on Physical Performance, Technical Activity and Injury Rate During Matches in a Professional Soccer Team. Br J Sports Med 2015;49: 390 - 394.

27. Hinman M. Factors Affecting Reliability of the Biodex Balance System: A summary of Four Studies. J Sport Rehab 2000:9: 240 - 252.

28. Lovell R, Knapper B, Small K. Physiological responses to SAFT90: a new soccer-specific match simulation. Coach Sports Sci 2008;3: 46. 
29. Sedliak M, Haverinen M, Hakkinen K. Muscle Strength, Resting Muscle Tone and EMG Activation in Untrained Men: Interaction Effect of time of Day and Test Order-Related Confounding Factors. J of Sports Med Phys Fit 2011;51: 560 - 570.

30. Brophy R, Silvers HJ, Gonzales T. Gender Influences: The Role of Leg Dominance in ACL Injury Among Soccer Players. Br J Sports Med 2010;44: 694 - 697.

31. Schmitz K, Arnold B. Intertester and Intratester Reliability of the J Sport Rehabil 1998;7: 95 - 101.

32. Yamada RKF, Arliani GC, Almeida GPL, Venturine AM, Dos Santos CV, Astur DC, Cohen M. The Effects of One-Half of a Soccer Match on the Postural Stability and Functional Capacity of the Lower Limbs in Young Soccer Players. Clinics 2012; 67: 1361 - 1364.

33. Franklin S, Grey MJ, Heneghan N, Bowen L, Li FX. Barefoot vs Common Footwear: A Systematic Review of Kinematic, Kinetic and Muscle Activity Differences during Walking. Gait Posture 2015;42: 230 - 239.

34. Cohen, J. Statistical power analysis for the behavioural sciences. 1988. $2^{\text {nd }}$ Edition. Hillsdale, NJ: Lawrence Earlbaum Associates.

35. Doyle TLA, Schilaty ND, Webster KE, Hewett TE. Time of Season and Game Segment is not Related to likelihood of Lower-Limb Injuries: A Meta-Analysis. Clin J Sports Med 2019; 0 (0): $1-9$.

36. Benjaminse A, Webster KE, Kimp A, Meijer M, Gokeler A. Revised Approach to the Role of Fatigue in Anterior Cruciate Ligament Injury Prevention: A Systematic Review with Meta Analyses. Sports Med 2019; 49: 565 - 586 
481

482

483

484

485

486

487

488

489

490

491

492

493

494

495

496

497

498

499

500

501

502

503

504

Table 1: Mean Directional Dynamic Stability Measures and Temporal Pattern Changes over a 72-hour Data Collection Period.

\begin{tabular}{|c|c|c|c|c|c|}
\hline \multirow[b]{2}{*}{$\begin{array}{c}\text { Dynamic Stability } \\
\text { Measures }\end{array}$} & \multicolumn{5}{|c|}{ Time Point } \\
\hline & Pre & Post & $+24 \mathrm{hrs}$ & +48 hrs & $+72 \mathrm{hrs}$ \\
\hline OSI & $2.42 \pm 1.07$ & $3.38 \pm 1.42$ & $4.22 \pm 1.58$ & $3.69 \pm 1.62$ & $3.00 \pm 1.48$ \\
\hline A-P & $1.89 \pm 1.01$ & $2.42 \pm 1.07$ & $3.06 \pm 1.06$ & $2.68 \pm 1.49$ & $2.24 \pm 1.29$ \\
\hline M-L & $\begin{array}{c}1.46 \pm 0.49 \\
(23 \%)\end{array}$ & $\begin{array}{c}2.07 \pm 0.69 \\
(14 \%)\end{array}$ & $\begin{array}{c}2.44 \pm 0.94 \\
(20 \%)\end{array}$ & $\begin{array}{c}2.14 \pm 0.85 \\
(20 \%)\end{array}$ & $\begin{array}{c}1.69 \pm 0.52 \\
(25 \%)\end{array}$ \\
\hline \multicolumn{6}{|c|}{$\begin{array}{l}\text { Note: }+24 \text { hrs }-24 \text { hours post fatigue; }+48 \text { hrs }-48 \text { hours post fatigue; }+72 \text { hrs }-72 \text { hours post fatigue. } \\
\% \text { indicates difference between A-P and M-L Stability Scores. } \\
\text { Data is presented as mean } \pm \text { SD. }\end{array}$} \\
\hline
\end{tabular}

$\%$ indicates difference between
Data is presented as mean \pm SD.

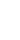


505 Figure Legends

506

507 Figure 1. Experimental set-up for Stabilometry testing.

508

509 Figure 2. The temporal pattern of recovery of OSI, A-P and M-L directional stability. 